\title{
The abnormality of the number of ribs misleading incorrect vertebral segment identification during lumbar intervention - Two cases report -
}

Received August 22, 2018

Revised October 4, 2018 Accepted October 22, 2018

\section{Corresponding author}

Ji-Seon Son, M.D., Ph.D. Department of Anesthesiology and Pain Medicine, Chonbuk National University Medical School and Hospital, 20 Geonji-ro, Deokjin-gu, Jeonju 54907, Korea

Tel: 82-63-250-1241

Fax: 82-63-250-1240

E-mail: sjs6803@jbnu.ac.kr

ORCID

https://orcid.org/0000-0002-6672-4576

\section{Jun Ho Lee, Ki-Jae Lee, Minjong Ki, Myungjong Kim, A Ram Doo, and Ji-Seon Son}

Department of Anesthesiology and Pain Medicine, Chonbuk National University Medical School and Hospital, Jeonju, Korea

\begin{abstract}
Background: For patients who have back pain or radiating pain, lumbar intervention should be performed at the correct lumbar segment that triggers pain. It is quite common for pain physicians to identify lumbar segments based upon the 12th pair of ribs to do an interventional procedure.

Case: We experienced two cases of rib number abnormality (absent 12th rib pair) that made the injection ineffective. In both cases, we had misidentified the lumbar segmentation due to rib abnormality. Although the procedure was performed properly, the clinical symptoms of the patient were not well correlated with the dermatome, and the diagnosis was delayed.
\end{abstract}

Conclusions: These cases suggest that rib counting is necessary for more accurate lumbar segmentation. If the expected effect has not appeared after intervention, rib numbers should be checked.

Keywords: Fluoroscopy; Injections; Lumbar vertebrae; Ribs.
Identification of the correct lumbar segment in order to perform interventional procedures for back pain patients with radiating pain in the lower extremities is very important. Injection at the wrong level leads to unwanted effects or no effect for the patients. Many pain physicians use fluoroscopy to identify the segments of the lumbar vertebrae, but it may cause confusion if the lumbar spine has anatomical deformities such as lumbarization or sacralization [1]. Thus, the fifth lumbar vertebra may be fused with the sacrum, or the sacrum may be separated to form the sixth lumbar vertebra. In these circumstances, many clinicians designate the $\mathrm{L} 1$ vertebra based upon the location of the 12th rib. The lowest rib attached costal facets would be identified as T12, and the vertebra beneath T12 identified as the first lumbar vertebra.
Jagannathan et al. [2] stated that the costal facets are reliable markers for T12 identification, and the lumbar segment can be fairly accurately identified in both the normal and the lumbarization groups. However, this method has a drawback, because up to $8 \%$ of normal children have only 11 pairs of ribs [3]. Here, we report a series of cases of interventional procedures on the wrong lumbar segment in patients whose 12th pair of ribs was absent.

\section{CASE REPORTS}

\section{Case 1}

A 70-year-old female visited our pain clinic complaining

This is an Open Access article distributed under the terms of the Creative Commons Attribution Non-Commercial License (http://creativecommons.org/licenses/by-nc/4.0) which permits unrestricted non-commercial use, distribution, and reproduction in any medium, provided the original work is properly cited.

Copyright (c) the Korean Society of Anesthesiologists, 2019 
of back pain with burning radiating pain on the left lower extremity persisting for two months. The radiating pain continued from the left buttock, posterolateral thigh to the left lateral calf with a tingling sensation. She rated her pain numerically as 4 out of 10 (0: no pain, 10: worst pain imaginable). Her sensations to light touch and pin prick were normal, her straight leg raising test was positive, and her dorsalis pedis arterial pulsation was intact. Lumbar spine magnetic resonance imaging (MRI) was recommended, and it found L2/3 diffuse disc bulging and L5 vertebral mild compression fracture with diffuse degenerative change of the L-spine.

Based upon the radiating pain of the lower limb, we thought there was involvement of the fifth lumbar nerve (L5), and attempted a transforaminal epidural steroidal injection (TFESI) in L5 (L5/S1). With the patient in prone position, we checked her lumbar segments by fluoroscopy based upon the 12th rib pair. The sixth lumbar spine, which indicates a lumbarization deformity, was observed from the fluoroscopic image. We performed transforaminal epidural steroid injection with a mixture solution of $0.1 \%$ ropivacaine $4 \mathrm{ml}$, dexamethasone $5 \mathrm{mg}$, and hyaluronidase $750 \mathrm{IU}$ at the left L5-6 counted from the 12th rib pair under C-arm fluoroscopy. Despite the steroidal injection, there was no significant symptom relief immediately after injection, and her symptoms did not improve over a week. She revisited two weeks after the injection. Because her symptom was not improved, and the shape of the L6 vertebra was similar to that of L5, we

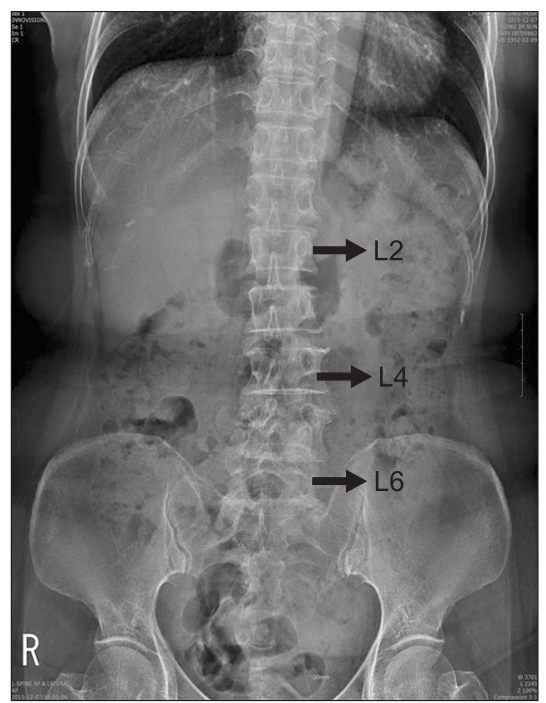

Fig. 1. The sixth lumbar vertebra (lumbarization) was observed by simple X-ray. suspected of lumbarization (Fig. 1). We counted the ribs by $\mathrm{C}$-arm fluoroscopy, and observed there were only 11 pairs of ribs. Therefore, TFESI was done one level lower than the previous injection. TFESI through the left L6/S1 intervertebral foramen was performed with the same mixture as before. In two weeks, the radiating pain to the lower limb was improved by up to $40 \%$, although the lower back pain still continued.

We have speculated the discordance the radiating pain pattern with nerve segments. Because we counted the lumbar segment based upon the lowest rib pair, the first lumbar vertebra was thought to be one level lower than the vertebra that has a rib pair. We wondered if the patient had a rib abnormality. To make sure, the rib series was taken by the radiologist and confirmed that she had only 11 pairs of ribs (Fig. 2). The first TFESI done at the L5/S1 level was L $4 / 5$ indeed, and the injection one level lower was at the real L5/S1 that we originally wanted.

\section{Case 2}

A 65-year-old female visited to our pain clinic due to severe pain on her left anterolateral thigh. She was being followed up for ovarian cancer, and the left paraaortic mass had metastasized to the L1 and L2 vertebrae. Her pain was rated numerically as 8 out of 10 . On physical examination, she had mild hypoesthesis and allodynia on the left anterolateral thigh, which corresponded with L2/3. She also complained about mild back pain and a tingling sensation in both calves,

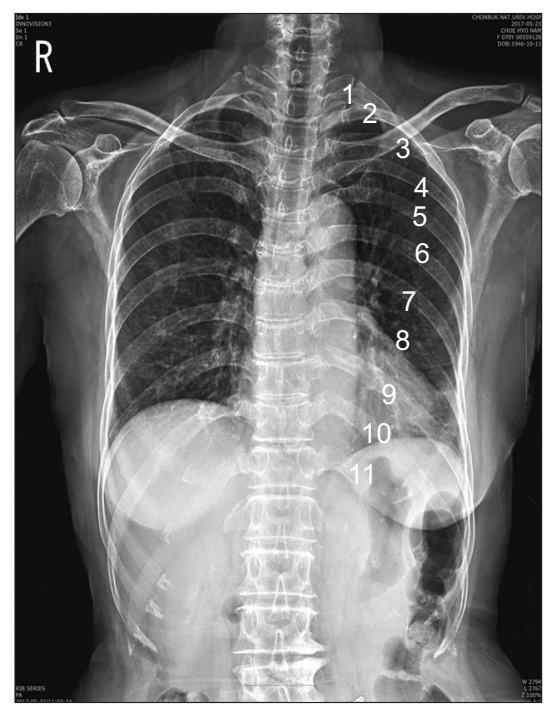

Fig. 2. The 12th pair of ribs was absent. 


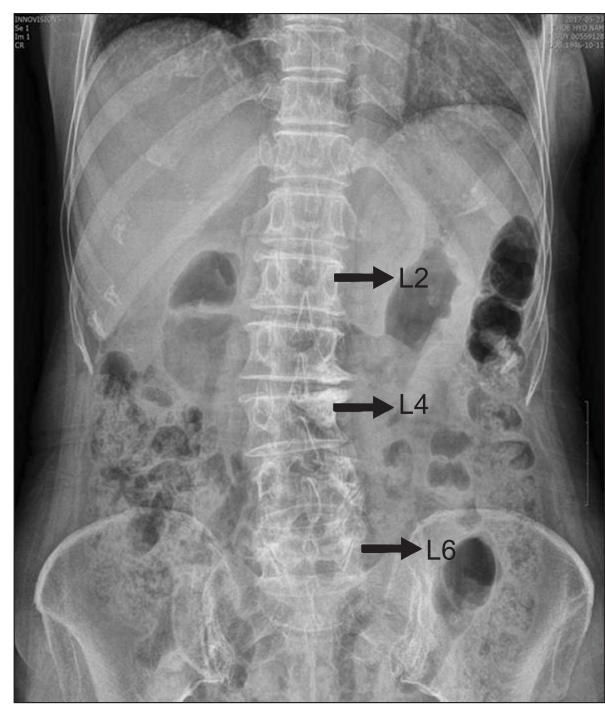

Fig. 3. The sixth lumbar vertebra (lumbarization) was observed by simple X-ray.

and the symptoms matched with dermatome of the S1 level. The authors suspected that the cause of the pain was the irritation of the L2 nerve root.

In order to perform a TFESI, we checked the lumbar segment based upon the lowest level of rib pair by fluoroscopy. The observed number of lumbar vertebrae was six, which meant a lumbarization deformity (Fig. 3). We did a TFESI with a mixture of $0.2 \%$ ropivacaine $4 \mathrm{ml}$ and dexamethasone $5 \mathrm{mg}$ at the left L2/3. Although the procedure was done without any difficulty, the pain was not relieved at all after the injection. Therefore, we presumed that the problem was not of central origin but was caused by peripheral nerve entrapment and suspected paresthetica meralgia. We observed the lateral femoral cutaneous nerve by means of ultrasonographic images, and used a mixture of $0.375 \%$ ropivacaine $10 \mathrm{ml}$ and triamcinolone $10 \mathrm{mg}$. The patient complained that the pain was still not relieved at all. Even a minimal blockade effect was not detected. Because we had experienced an abnormality of the number of ribs, we again counted the ribs by fluoroscopy and found that the patient also had 11 pairs of ribs. Therefore, we performed a TFESI at one level lower, through the actual L2/3 vertebral foramen. Right after the TFESI, significant pain relief was observed; so the segmentation and injection had been properly performed. As in the previous case, the first injected site at $\mathrm{L} 2 / 3$ was actually $\mathrm{Ll} / 2$.

Unfortunately, the effect did not persist too long after we followed up the patient for two weeks. Gabapentin $400 \mathrm{mg}$ three times a day was given, but pain relief was not apparent. Although it is reasonable to regard compression fracture with facet joint syndrome in clinical features, we carried out an additional examination because she was a cancer patient and reevaluated the left paraaortic mass by PET-CT. The size of the mass had increased $(3.4 \mathrm{~cm} \rightarrow 5.3 \mathrm{~cm})$, and we found that the mass had invaded the L1, L2 vertebral body and left psoas muscle. She was transferred to the department of obstetrics and gynecology, and concurrent chemotherapy and radiotherapy was conducted. Her pain was decreased in a week.

\section{DISCUSSION}

Lumbosacral transitional vertebrae are common spinal anomalies defined as sacralization or lumbarization. The prevalence of those anomalies has been reported as up to $30 \%$ [4]. Also, symptoms can originate from many segments, and physical examination may be confused in many cases [5]. Because the spinal anomalies differ morphologically, it is challenging but important to identify the correct lumbar segment as the actual site of the pain source. Many pain physicians use fluoroscopy to identify the segment of the lumbar vertebrae, but it may cause confusion if the lumbar spine has those lumbosacral transitional vertebrae. Inaccurate segment identification may lead to intervention at unintended levels. Injecting at the wrong segment is not only ineffective for the patient but also affects the future diagnostic plan, and may delay other therapeutic modalities, such as surgery or chemotherapy.

Furman et al. [6] summarized the method of identifying vertebral levels. Vertebral segments are identified by counting down from the $\mathrm{C} 2$ vertebra using imaging studies. Also, anatomic landmarks, such as transverse processes of the lowest lumbar vertebra and iliac crest, are used for identifying vertebral levels. If the segments are not clearly identified by the methods mentioned above, vertebrae can be defined by counting down from the T12 vertebra, where the lowest rib originates. However, identified abnormalities and the patient's clinical findings should be correlated, because none of the identification methods is $100 \%$ accurate. Many clinicians designate the $\mathrm{L} 1$ vertebra by locating the 12 th rib. The costal facets that attach ribs are observed at the $\mathrm{T} 12$ vertebrae in $96.9 \%$ of normal patients and $91.7 \%$ of lumbarization patients. Thus, costal facets can be used as a reliable marker for 
the T12 vertebrae [2]. However, some rib-number anomalies have been reported, especially in pediatric patients. In a study, an abnormal number of ribs was observed in $8 \%$ of fetuses, and most of those anomalies had 11 pairs of ribs [3]. Although normal variants are usually clinically insignificant, ribs can differ in number, size, and shape [7]. As far as we know, there has been no report of injections in undesired locations due to absence of ribs in adult population. We recommend counting vertebrae from $\mathrm{T} 1$ if a patient does not have a prior image or if the intervention does not correlate with the symptoms of the patient.

In the first case, we had misidentified the 11th ribs as the 12th ribs, and had no effect from the intervention. Although the procedure was performed properly, the clinical symptoms of the patient were not well correlated with the dermatome [8]. The second case was also an example of misidentification of lumbar segments because of rib-number abnormality, but the effect was insufficient even after proper intervention following rib count. Thus, wrong segmentation delayed the diagnosis of the increased size of the mass. Also, the diagnostic blockade should be performed on the same day using two local anesthetics with different half-lives, such as lidocaine and bupivacaine, and should be diagnosed by the time they affect the sensory nerve segments.

In conclusion, we recommend counting the number of ribs by C-arm fluoroscopy or X-ray to make sure the spinal segment is accurate when the intervention has been performed correctly. If the expected effect has not appeared after intervention, rib numbers should be checked.

\section{CONFLICTS OF INTEREST}

No potential conflict of interest relevant to this article was reported.

\section{ORCID}

Jun Ho Lee: https://orcid.org/0000-0002-9424-8589

Ki-Jae Lee: https://orcid.org/0000-0002-2591-455X

Minjong Ki: https://orcid.org/0000-0001-9959-7908

Myungjong Kim: https://orcid.org/0000-0002-0031-2253

A Ram Doo: https://orcid.org/0000-0003-1310-790X

\section{REFERENCES}

1. Jain A, Agarwal A, Jain S, Shamshery C. Bertolotti syndrome: a diagnostic and management dilemma for pain physicians. Korean J Pain 2013; 26: 368-73.

2. Jagannathan D, Indiran V, Hithaya F, Alamelu M, Padmanaban S. Role of anatomical landmarks in identifying normal and transitional vertebra in lumbar spine magnetic resonance imaging. Asian Spine J 2017; 11: 365-79.

3. Khodair SA, Hassanen OA. Abnormalities of fetal rib number and associated fetal anomalies using three dimensional ultrasonography. Egypt J Radiol Nucl Med 2014; 45: 689-94.

4. Konin GP, Walz DM. Lumbosacral transitional vertebrae: classification, imaging findings, and clinical relevance. AJNR Am J Neuroradiol 2010; 31: 1778-86.

5. Ahn K. Segmental palpation for radiculopathy. Anesth Pain Med 2011; 6: 101-8.

6. Furman MB, Wahlberg B, Cruz EJ. Lumbosacral transitional segments: an interventional spine specialist's practical approach. Phys Med Rehabil Clin N Am 2018; 29: 35-48.

7. Glass RB, Norton KI, Mitre SA, Kang E. Pediatric ribs: a spectrum of abnormalities. Radiographics 2002; 22: 87-104.

8. Ladak A, Tubbs RS, Spinner RJ. Mapping sensory nerve communications between peripheral nerve territories. Clin Anat 2014; 27: 681-90. 